
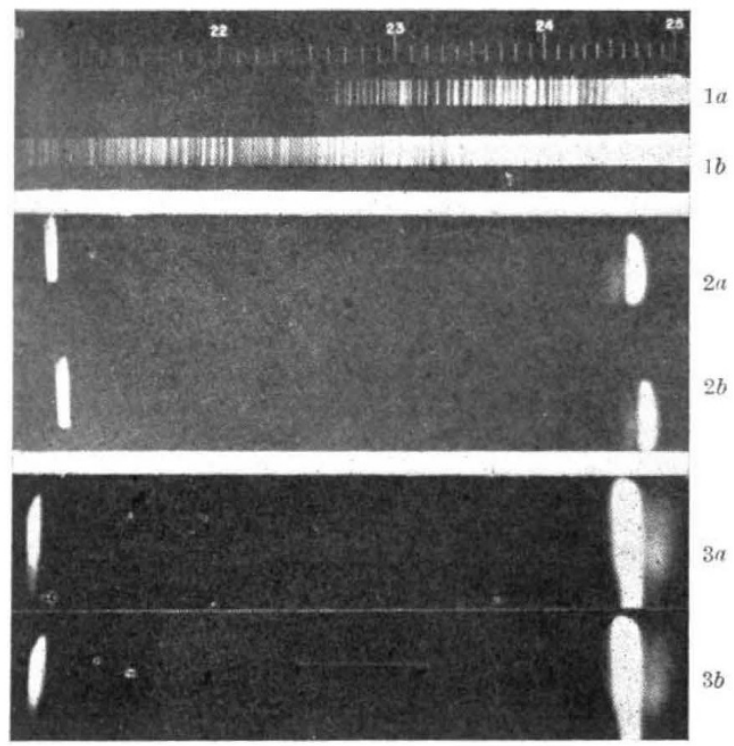

1a: ABSORPTION SPECTRUM OF DIAMOND (N.C. 125).

$1 b$ : SPECTRUM OF THE IRON ARC.

$2 a$ and $2 b$ : PHOTOGRAPHS OF THE LAUE AND DYNAMIO (111) X-RAY REFLEXIONS WITH THE DIAMOND AT $600^{\circ} \mathrm{C}$. AND AT ROOM TEMPERATURE RESPECTIVELY.

$3 a$ AND $3 b:$ SIMILAR PHOTOGRAPHS TAKEN WITH THE DIAMOND AT ROOM TEMPERATURE AND AT LIQUID AIR TEMPERATURE RESPECTIVELY.

shown conclusively that the diamonds, which are throughout of the ultra-violet opaque type $e^{5,8}$, possess a high degree of crystal perfection. On the other hand, diamonds of the ultra-violet transparent type have a variable crystal spacing?, accompanied by a pronounced lamellar birefringence. It is not surprising, therefore, that the dynamic X-ray reflexions given by this type of diamond do not exhibit the same sharpness and intensity as those given by diamonds of the ultra-violet opaque kind. Indeed, even the Laue reflexions given by these diamonds often show obvious irregularities. The fact that only the most intense of the three quantum reflexions indicated by the Raman-Nath formula is observed with diamonds of the ultra-violet transparent type is also readily intelligible when the above considerations are taken into account. We have further verified that, within the limits of the error set by the imperfection of the observed reflexions, their positions agree with those given by the Raman-Nath formula.

The objections which Mrs. Lonsdale has raised against the interpretation of the X-ray reflexions observed with diamond given by the Bangalore workers are thus believed to be without experimental foundation.

R. S. Krishnan.

G. N. RAMACHANDRAN.

Physics Department,

Indian Institute of Science,

Bangalore.

Jan. 5.

${ }^{1}$ Raman, C. V., and Nilakantan, P., Curr. Sci. 9, 165 (1940).

'Raman, C. V., Proc. Ind. Acad. Sci., A, 14, 317, 332 (1941). Raman, C. V., and Nilakantan, P., Proc. Ind. Acad. Sci., A, 14, 356 (1941)

'Raman, C. V., and Nilakantan, P., Nature, 147, 118 (1941).

'Lonsdale, K., Proc. Roy. Soc., A, 179, 315 (1942).

${ }^{\circ}$ Raman, C. V., and Rendall, G. R., Proc. Ind. Acad. Sci., A, 19, 265 (1944).

- Ramachandran, G. N., Proc. Ind. Acad. Sci., 20, 245 (1944).

'Krishnan, R. S., Proc. Ind. Acad. Sci., 19, 298 (1944).

\section{Thermomechanical Effect in Liquid Helium II}

THE peculiar properties of liquid helium II have been the subject of many experimental and theoretical investigations during recent years. The characteristic features of liquid helium II are its superfluidity (viscosity less than one billionth of that of water) and the thermomechanical effect. The superfluidity has been explained by F. London ${ }^{1}$ and L. Tisza ${ }^{2}$ by assuming helium II to be in a state of Bose-Einstein degeneracy, a part of the atoms constituting the condensed phase. In a previous communication ${ }^{3}, \mathrm{I}$ have given a theory of the surface-flow of liquid helium II in the form of thin mobile films, and my purpose now is to discuss the thermomechanical effect in helium II assuming the latter to be in the state of Bose-Einstein degeneracy.

Landau ${ }^{4}$ has given a different theory based on the model of a 'quantum liquid'. H. London ${ }^{5}$ has given a thermodynamical discussion of the thermomechanical effect and has shown (taking the London-Tisza model of helium II) that

$$
(d p / d t)_{\max .}<J \rho \varphi,
$$

where $\varphi$ is the entropy in cal. per gm., $J$ is the mechanical equivalent of heat and $(d p / d t)_{\max }$. is an upper limit to the reaction pressure per degree. The expression for the thermomechanical effect can also be obtained as follows :

The pressure and energy per unit volume in a BoseEinstein degenerate gas are connected by the relation

$$
p_{-}=C \Gamma(S) \zeta(S+1)(k T)^{S+1}=\frac{E_{-}}{S \tilde{V}} .
$$

$S$ is defined by the equation

$$
N(\varepsilon) d \varepsilon=\frac{V C \varepsilon^{S-1} d \varepsilon}{1 / A \cdot e^{C / k T-1}} \quad . \quad . .
$$

$N(\varepsilon) d \varepsilon$ denotes the number of particles having kinetic energies between $\varepsilon$ and $\varepsilon+d \varepsilon$.

As remarked by $H$. London, $S$ is to be taken equal to 5 in order that the observed discontinuity in the (constant volume) specific heat at the $\lambda$-point may agree with the theoretical value.

From (1) we have

$$
\frac{(d p)}{(d t)}=\frac{I}{S} \frac{d(E / V)}{d t}=\frac{\rho C_{v}}{S}
$$

where $C_{v}$ is the specific heat at constant volume (per unit mass). The above relation is roughly in accord with observations. For temperatures $1 \cdot 393^{\circ} \mathrm{K}$. and $1 \cdot 241^{\circ} \mathrm{K}$., $C_{v}$ is equal to $0 \cdot 311$ and $0 \cdot 174$ respectively.

Thus we have for $(d p / d t)$ at $1 \cdot 393^{\circ} . \mathrm{K}$. and $1 \cdot 24^{\circ} \mathrm{K}$., the values $3.65 \times 10^{5}$ and $2.04 \times 10^{5}$, which may be compared with the observed values $1.7 \times 10^{5}$ and $0.6 \times 10^{5}$ respectively, corresponding to the temperatures given above.

Baroda College,

D. V. Gogate.

Baroda.

Dec. 17.

${ }^{1}$ London, F., Phys. Rev., 54, 947 (1938).

2 Tisza, Nature, 141, 913 (1938).

Gogate and Rai, Nature, 153, 342 (1944)

- Landau, L., Phys. Rev., 60, 356 (1941).

${ }^{5}$ London, H., Proc. Roy. Soe, A, 171, 484 (1939). 